\title{
Editorial: Orofacial Pain, Bruxism, and Sleep
}

\author{
Mieszko Wieckiewicz ${ }^{1 *}$ and Efraim Winocur ${ }^{2}$ \\ ${ }^{1}$ Department of Experimental Dentistry, Wroclaw Medical University, Wrocław, Poland, ${ }^{2}$ Department of Oral Rehabilitation, \\ The Maurice and Gabriela Goldschleger School of Dental Medicine, Tel Aviv University, Tel Aviv, Israel
}

Keywords: orofacial pain, sleep bruxism, awake bruxism, sleep, temporomandibular disorders

\section{Editorial on the Research Topic}

\section{Orofacial Pain, Bruxism, and Sleep}

The task force on taxonomy of the International Association for the Study of Pain (IASP) defines pain as "An unpleasant sensory and emotional experience associated with actual or potential tissue damage, or described in terms of such damage" (1). Furthermore, IASP says that orofacial pain is a frequent form of pain perceived in the face and/or oral cavity. It may be caused by diseases or disorders of regional structures, dysfunction of the nervous system, or through referral from distant sources. The orofacial pain is a serious global problem which affects $\sim 20 \%$ of human population each year. According to the current approach (2), bruxism is considered as two different behaviors observed during sleep and wakefulness, respectively, and the single definition for bruxism has been

OPEN ACCESS

Edited by:

Sabina Cevoli,

IRCCS Institute of Neurological Sciences of Bologna (ISNB), Italy

Reviewed by: Andreas Gantenbein, RehaClinic, Switzerland Lanfranco Pellesi, Danish Headache Center, Rigshospitalet, Denmark

${ }^{*}$ Correspondence:

Mieszko Wieckiewicz m.wieckiewicz@onet.pl

Specialty section

This article was submitted to Headache Medicine and Facial Pain, a section of the journal Frontiers in Neurology

Received: 11 October 2019 Accepted: 15 May 2020

Published: 10 June 2020

Citation: Wieckiewicz M and Winocur E (2020) Editorial: Orofacial Pain, Bruxism, and Sleep. Front. Neurol. 11:555. doi: 10.3389/fneur.2020.00555 replaced by two separate definitions: (1) Sleep bruxism is a masticatory muscle activity during sleep that is characterized as rhythmic (phasic) or non-rhythmic (tonic) and is not a movement disorder or a sleep disorder in otherwise healthy individuals. (2) Awake bruxism is a masticatory muscle activity during wakefulness that is characterized by repetitive or sustained tooth contact and/or by bracing or thrusting of the mandible and is not a movement disorder in otherwise healthy individuals.

Even though the etiopathogenesis of bruxism is not fully understood, many different factors are believed to be associated with this muscular activity. An increasing number of evidence suggests a relationship between bruxism and other disorders, conditions or systemic diseases, including sleep breathing disorders, uncontrolled limbs movements during sleep, reflux disease, neurological disorders and orofacial pain $(3,4)$. This Research Topic aimed to determine the nature of sleep bruxism and its types, as well as investigate the relationship between sleep bruxism and orofacial pain as well as different general health diseases, conditions and disorders.

Nine articles had been included in this Research Topic.

Three published articles are concerning: the prevalence of orofacial and general pain related to muscular temporomandibular disorders (Kuć et al.), and prevalence of insomnia among patients with orofacial pain (Cruz et al.), and epidemiology of awake and sleep bruxism among adolscents (Winocur et al.).

Three articles are related to diagnostic methods in a group of people with increased activity of masticatory muscles (Osiewicz et al.; Szyszka-Sommerfeld et al.; Zani et al.). Furthermore, it should be emphasized that two from mentioned three papers describe the new diagnostic method of awake bruxism i.e., ecological momentary assessment and intervention principles for the study of awake bruxism behaviors. Next two papers assessed relationship between sleep bruxism and headache (Martynowicz et al.), as well as sleep bruxism and TMD-related pain (Smardz et al.).

A single article describes the nociceptive effect of platelet-rich plasma intramuscular injections in myofascial pain of masseter muscles (Nitecka-Buchta et al.). 
Published articles confirm how difficult clinical issues are related to impaired function of the orofacial structures. At the same time, the described studies indicate that the etiology of complaints reported by patients can be very compound. Thus, it requires an objective diagnostic approach and personalized therapeutic strategies. Therefore, the approach to sufferers should be holistic based on broad evidence-based knowledge from various fields of medicine.

\section{REFERENCES}

1. International Association for the Study of Pain (IASP). IASP Terminology. Available online at: https://www.iasp-pain.org/Education/Content.aspx? ItemNumber $=1698 \#$ Pain

2. Lobbezoo F, Ahlberg J, Raphael KG, Wetselaar P, Glaros AG, Kato T, et al. International consensus on the assessment of bruxism: report of a work in progress. J Oral Rehabil. (2018) 45:837-44. doi: 10.1111/joor.12663

3. Khoury S, Carra MC, Huynh N, Montplaisir J, Lavigne GJ. Sleep bruxism-tooth grinding prevalence, characteristics and familial aggregation: a large crosssectional survey and polysomnographic validation. Sleep. (2016) 39:2049-56. doi: $10.5665 /$ sleep. 6242

4. Carra MC, Huynh N, Lavigne G. Sleep bruxism: a comprehensive overview for the dental clinician interested in sleep medicine.
Editors strongly encourage potential readers to look at the articles.

\section{AUTHOR CONTRIBUTIONS}

All authors listed have made a substantial, direct and intellectual contribution to the work, and approved it for publication.

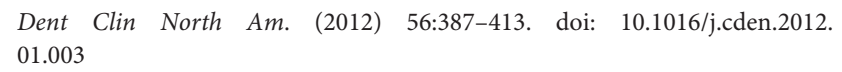

Conflict of Interest: The authors declare that the research was conducted in the absence of any commercial or financial relationships that could be construed as a potential conflict of interest.

Copyright (c) 2020 Wieckiewicz and Winocur. This is an open-access article distributed under the terms of the Creative Commons Attribution License (CC BY). The use, distribution or reproduction in other forums is permitted, provided the original author(s) and the copyright owner(s) are credited and that the original publication in this journal is cited, in accordance with accepted academic practice. No use, distribution or reproduction is permitted which does not comply with these terms. 